\title{
Nonlinear dynamics in experimental devices with compressed/expanded surfactant monolayers
}

\author{
M Higuera, J M Perales and J M Vega
}

\begin{abstract}
A theory is provided for a common experimental set up that is used to measure surface properties in surfactant monolayers. The set up consists of a surfactant monolayer (over a shallow liquid layer) that is compressed/expanded in a periodic fashion by moving in counter-phase two parallel, slightly immersed solid barriers, which vary the free surface area and thus the surfactant concentration. The simplest theory ignores the fluid dynamics in the bulk fluid, assuming spatially uniform surfactant concentration, which requires quite small forcing frequencies and provides reversible dynamics in the compression/expansion cycles. In this paper, we present a long-wave theory for not so slow oscillations that assumes local equilibrium but takes the fluid dynamics into account. This simple theory uncovers the physical mechanisms involved in the surfactant behavior and allows for extracting more information from each experimental run. The conclusion is that the fluid dynamics cannot be ignored, and that some irreversible dynamics could well have a fluid dynamic origin.
\end{abstract}

(Some figures may appear in colour only in the online journal)

\section{Introduction}

Surfactant monolayers are relevant in a wide variety of scientific and technological fields. On the scientific side, the dynamics of fluid systems with interfaces are relevant (Miller and 
Scriven 1968) in a variety of disciplines ranging from chemical, soft matter and polymer physics to colloid and interface science, biology, biochemistry and biophysics. On the technological side, surfactants are of interest (Sagis 2011) in connection with, e.g. the mechanical, chemical, agricultural, pharmaceutical and medical industries. In general, surface rheology cannot be ignored in microfluidics and is essential in understanding coating and drying processes, thin film stability and dynamics (Couder et al 1989), and the formation and dynamics of foams (Langevin and Bergeron 1996). Analyzing surfactant monolayers requires knowledge of surface properties such as Marangoni elasticity, surfactant diffusion and surface (dilatational and shear) viscosity, whose measurement has received continuous attention in recent decades. Surfactant dynamics may involve anisotropy and viscoelastic effects, solubility in the bulk phase and other non-equilibrium phenomena (Fainerman et al 2010) depending on various spatio-temporal scales, which makes both understanding the phenomena and designing/predicting/explaining experimental measurements fairly subtle.

A common experimental set up, which is used to measure surface properties that manifest themselves in large time scales consists in a shallow liquid layer whose free surface is subject to periodic compression/expansion. This is done by moving in counter-phase two (slightly immersed) solid barriers, which vary the enclosed free surface area in a controlled fashion. The time-dependent surface tension is measured at the symmetry axis of the monolayer and the resulting time series are used to extract information about the monolayer dynamics. The forcing frequency is quite small, intending spatially uniform surfactant concentration, trying to avoid coupling with the fluid dynamics in the bulk fluid and non-equilibrium phenomena. In this case, the surfactant concentration is calculated as inversely proportional to the known area between the solid barriers, and thus the time series of the surfactant concentration is readily obtained. If local equilibrium is assumed, then the system should exhibit reversible dynamics in the compression/expansion cycles. In particular, the experiments carried out by Hilles et al (2007) show that the oscillatory motions of the barriers lead to hysteresis cycles, whose area increases with the strain amplitude; see also the experimental results by Kato and Kawaguchi (2012) and Kobayashi and Kawaguchi (2013). This irreversible behavior is sometimes attributed to non-equilibrium effects related to physico-chemical properties of the surfactant, like for example phase-changes in the monolayer. However, in all these works the fluid dynamics of the bulk liquid is systematically ignored. In our opinion, this point is essential to build a solid ground for understanding these phenomena. In other words, uncovering the role of the fluid dynamics and elucidating to what extend the fluid dynamics can explain some of the observed phenomena could be a first step, before looking for more complex formulations that include non-equilibrium effects.

The goal of this paper is to develop a theory for not so slow oscillations that provides a spatially non-uniform surfactant concentration, intending to uncover the physical mechanisms involved in the surfactant dynamic behavior. We will take the fluid dynamics into account using a long-wave, lubrication approximation that takes advantage of the fact that the distance between the solid barriers is large compared to the fluid layer depth. The resulting asymptotic model is still fairly computationally expensive, since the hydrodynamics is fully coupled with the monolayer dynamics. Thus, it will be further simplified in a distinguished limit in which the forcing frequency is small compared to the inverse of the viscous time based on the container depth, but not so small as to impose spatially uniform surfactant concentration. In this intermediate limit, the fluid dynamics in the bulk is slaved to the surfactant concentration distribution due to Marangoni elasticity. The fluid dynamics can then be solved in closed form and a nonlinear diffusion equation is obtained for the surfactant concentration dynamics.

With these ideas in mind, the remainder of the paper is organized as follows. The problem is first formulated in section 2, where the typical experimental values of the involved 


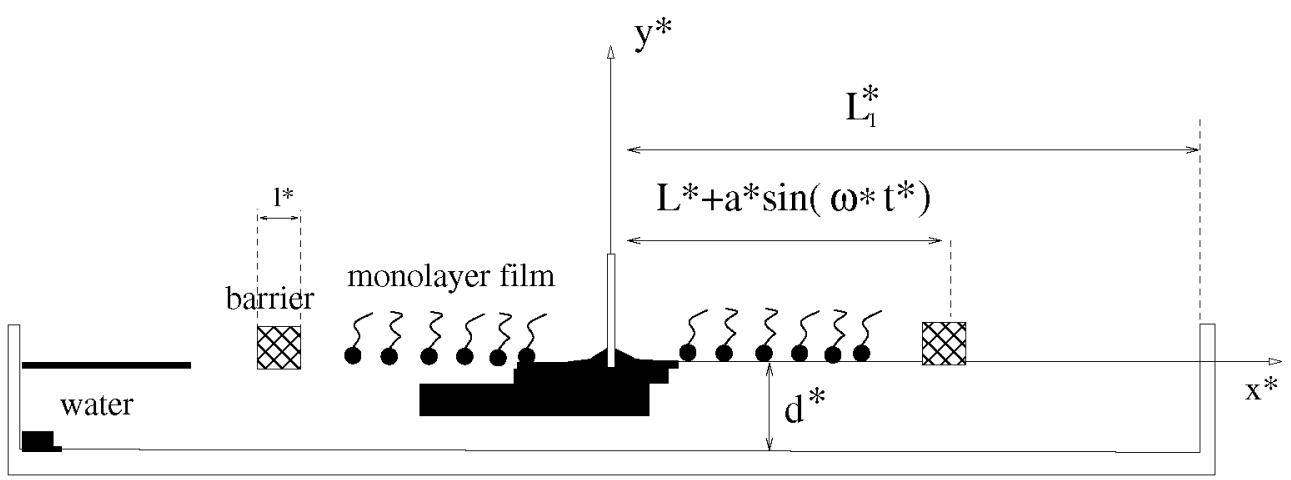

Figure 1. Sketch of a two-dimensional Langmuir trough.

non-dimensional parameters are accounted for. The long-wave approximation will be developed in section 3, where the above-mentioned distinguished limit will also be considered. The typical expected behaviors are discussed in section 4 . The paper ends with some concluding remarks, in section 5 .

\section{Formulation}

We consider a horizontal, rectangular container of horizontal size $2 L_{1}^{*} \times 2 L_{2}^{*}$, filled with a liquid layer of unperturbed depth $d^{*} \ll L_{1}^{*} \sim L_{2}^{*}$; the superscript ${ }^{*}$ is used hereinafter to denote dimensional quantities. The system is described using a Cartesian coordinate system (see figure 1) with the $x^{*}$ and $z^{*}$ axes along the symmetry axes of the unperturbed free surface, and the $y^{*}$ axis pointing upward. Two horizontal solid barriers, of width $\ell^{*} \ll L_{1}^{*}$, parallel to the $z^{*}$ axis are symmetrically moved in counterphase, in the $x^{*}$ direction, in a periodic fashion. The barriers are slightly immersed in the liquid layer, intending to minimize the effect of their motion on the bulk fluid. A surfactant is spread initially in that part of the free surface between the solid barriers and allowed to relax to the spatially uniform state before initiating the motion of the solid barriers. If the capillary menisci near the lateral walls are small compared to $L_{2}^{*}$, as we assume hereafter, a reasonable assumption is that all subsequent states of the system are independent of the transversal coordinate $z^{*}$, obtaining a two dimensional formulation.

Surface tension $\sigma$ in the central region between the solid barriers is assumed to depend on the surfactant concentration $c^{*}$ in such a way that $\sigma$ monotonously decreases as $c^{*}$ increases. In fact, this dependence will be expressed below in terms of the so-called surface pressure, defined as

$$
\Pi^{*}\left(c^{*}\right)=\sigma(0)-\sigma\left(c^{*}\right) \geqslant 0 .
$$

Surfactant concentration gradients promote a tangential stress that is proportional to the gradient of the surface pressure, namely to

$$
\nabla \Pi^{*}=M^{*}\left(c^{*}\right) \nabla c^{*} \quad \text { with } M^{*}\left(c^{*}\right)=\mathrm{d} \Pi^{*} / \mathrm{d} c^{*},
$$

where $M^{*}$ is the Marangoni elasticity, which is usually unimodal.

A typical experimental plot of $\Pi^{*}$ and $M^{*}$ versus the surfactant concentration is provided in figure 2 (left), where the three distinguished regimes are indicated. In the diluted regime, for very low surfactant concentrations, $\Pi^{*}$ shows first a linear behavior, and then a power law 

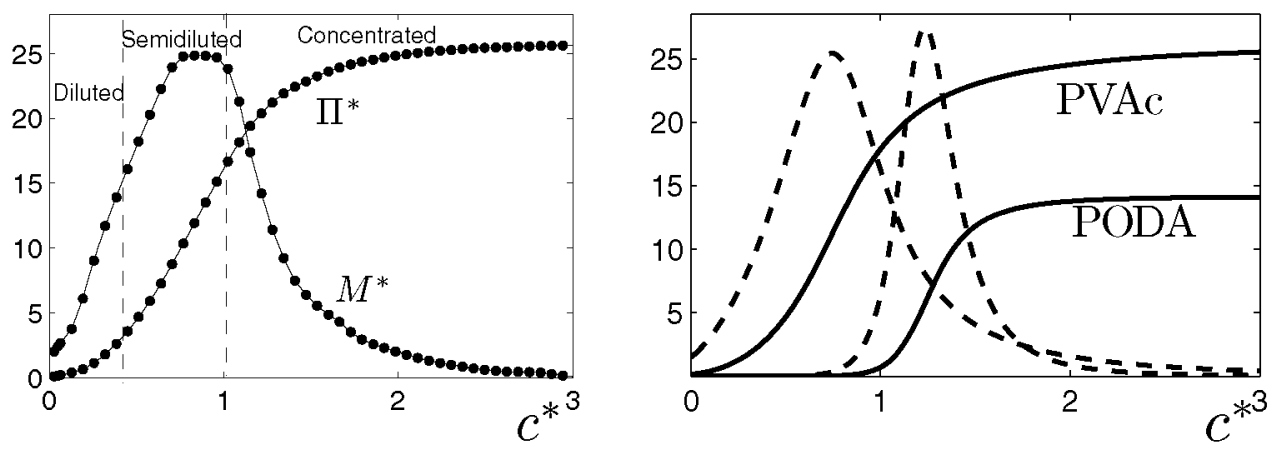

Figure 2. Marangoni elasticity $M^{*}$ (in $\mathrm{mNmmg}^{-1}$ and surface pressure $\Pi^{*}$ (in $\mathrm{mN} \mathrm{m}^{-1}$ ) versus $c^{*}$ (in $\mathrm{mg} \mathrm{m}^{-2}$ ). Left: experimental curves for poly(vinyl acetate), PVAc, with the three regimes indicated; courtesy of R G Rubio. Right: analytically adapted curves for PVAc and poly(octadecyl acrylate), PODA, as indicated, plotting $M^{*}$ and $\Pi^{*}$ with dashed and solid lines, respectively.

behavior matches the semi-diluted regime. The concentrated regime corresponds to higher surfactant concentration values, where the Marangoni elasticity decreases as the surfactant concentration increases. The surfactant may exhibit some plastic behavior (excluded below) in this regime, at least for some surfactants (Hilles et al 2006). Nevertheless, this could not always be the case and thus this regime will also be considered in the context of this paper. Figure 2 (right) shows the analytical approximations for $\Pi^{*}$ and $M^{*}$ that will be used below for poly(vinyl acetate), PVAc, and also for poly(octadecyl acrylate), PODA, which shows both a steeper behavior in the semidiluted regime and a flatter behavior at both diluted and concentrated regimes, as can be seen in the experimental curves obtained by Hilles et al (2007) (see figure 2(a) in that paper).

In order to formulate the problem, $d^{*}, \mu /\left(\rho d^{*}\right), \omega^{*-1}, \mu^{2} /\left(\rho d^{* 2}\right)$ and $c_{0}^{*}$ are used as units to non-dimensionalize length, velocity, time, pressure and surfactant concentration, respectively, where $\rho$ and $\mu$ are the density and the viscosity in the bulk liquid, $\omega^{*}$ is the frequency of the barriers motion and $c_{0}^{*}$ is a typical surfactant concentration value that will be chosen below (depending on the regime). Thus, the non-dimensional variables and parameters are defined as

$$
\begin{aligned}
& (x, y)=\left(x^{*}, y^{*}\right) / d^{*}, \quad\left(L, L_{1}, a\right)=\left(L_{0}^{*}, L_{1}^{*}, a^{*}\right) / d^{*}, \quad f=f^{*} / d^{*}, \\
& t=\omega^{*} t^{*}, \quad(u, v)=\rho d^{*}\left(u^{*}, v^{*}\right) / \mu, \quad p=\rho d^{* 2} p^{*} / \mu^{2}, \quad c=c^{*} / c_{0}^{*},
\end{aligned}
$$

where $a^{*}$ is the amplitude of the barriers motion, $f^{*}$ is the vertical elevation of the free surface, $u^{*}$ and $v^{*}$ are the horizontal and vertical velocity components, respectively, $p^{*}$ is the pressure and $c^{*}$ is the surfactant concentration. The resulting non-dimensional continuity and Navier-Stokes equations are

$$
\begin{aligned}
& \partial_{x} u+\partial_{y} v=0 \\
& \omega \partial_{t} u+u \partial_{x} u+v \partial_{y} u=-\partial_{x} p+\partial_{x x}^{2} u+\partial_{y y}^{2} u \\
& \omega \partial_{t} v+u \partial_{x} v+v \partial_{y} v=-\partial_{y} p+\partial_{x x}^{2} v+\partial_{y y}^{2} v,
\end{aligned}
$$

where $\partial_{t}, \partial_{x}, \partial_{y}, \partial_{x x}, \ldots$ denote hereafter partial derivatives and the dimensionless frequency $\omega$ is as defined below, in equation (15). These equations apply in the spatial region in between 
of the solid barriers, which is

$$
-L X_{\mathrm{b}}(t)<x<L X_{\mathrm{b}}(t), \quad-1<y<f,
$$

where

$$
X_{\mathrm{b}}=1+a \sin t
$$

is a half of the non-dimensional distance between the barriers. In order to facilitate using different periodic and aperiodic laws for the barriers motion, the theory below will be formulated for general $X_{\mathrm{b}}$.

The boundary conditions at the solid barriers, $x= \pm L X_{\mathrm{b}}(t)$, are subtle and will only be considered in the following section, under some additional simplifying assumptions. The boundary conditions at the bottom of the container account for no-slip, namely

$$
u=v=0 \quad \text { at } y=-1,
$$

and the boundary conditions the free surface result from kinematic compatibility and equilibrium of tangential and normal stresses, namely

$\omega \partial_{t} f+u \partial_{x} f=v$,

$\partial_{y} u+\partial_{x} v+\left[2 \partial_{y} v-2 \partial_{x} u-\left(\partial_{y} u+\partial_{x} v\right) \partial_{x} f\right] \partial_{x} f=-\mathcal{P} \partial_{x} \Pi(c) / \sqrt{1+\left|\partial_{x} f\right|^{2}}$,

$p-\mathcal{G} f+\partial_{x}\left[\frac{[\mathcal{S}-\mathcal{P} \Pi(c)] \partial_{x} f}{\left(1+\left|\partial_{x} f\right|^{2}\right)^{1 / 2}}\right]=2 \frac{\partial_{y} v-\left(\partial_{y} u+\partial_{x} v\right) \partial_{x} f+\partial_{x} u\left(\partial_{x} f\right)^{2}}{1+\left|\partial_{x} f\right|^{2}}$,

$\omega \partial_{t}\left[\sqrt{1+\left|\partial_{x} f\right|^{2}} c\right]+\partial_{x}\left[\left(u-v \partial_{x} f\right) c\right]=\mathcal{D} \partial_{x}\left[\frac{D(c) \partial_{x} c}{\sqrt{1+\left|\partial_{x} f\right|^{2}}}\right]$

at $y=f$, where we have neglected surface viscous effects in (11) and for convenience, we have also added the surfactant concentration equation (13). Note that (to facilitate interpretation of the results, see below) we are retaining surfactant diffusion, which is likely to be negligible compared to the retained effects.

The non-dimensional surface pressure $\Pi$ and the surfactant diffusion $D$ are defined in terms of their dimensional counterparts as

$$
\Pi(c)=\Pi^{*}\left(c_{0}^{*} c\right) / \Pi^{*}\left(c_{0}^{*}\right), \quad D(c)=D^{*}\left(c_{0}^{*} c\right) / D^{*}\left(c_{0}^{*}\right),
$$

and the various non-dimensional parameters appearing in (5), (6), (10)-(12) are

$$
\begin{aligned}
& \omega=\rho\left(d^{*}\right)^{2} \omega^{*} / \mu, \quad \mathcal{G}=\rho^{2} g d^{* 3} / \mu^{2}, \quad \mathcal{S}=\rho \sigma_{0} d^{*} / \mu^{2}, \\
& \mathcal{P}=\rho d^{*} \Pi^{*}\left(c_{0}\right) / \mu^{2}, \quad \mathcal{D}=\rho D^{*}\left(c_{0}^{*}\right) / \mu .
\end{aligned}
$$

For typical terrestrial $\left(g=9.8 \times 10^{2} \mathrm{~cm} \mathrm{~s}^{-2}\right.$ ) conditions, using a bulk liquid such as water $\left(\rho \sim 1 \mathrm{~g} \mathrm{~cm}^{-3}, \mu \sim 0.01 \mathrm{~g} \mathrm{~cm}^{-1} \mathrm{~s}^{-1}\right)$, and a experimental set up with $d^{*} \sim 0.5 \mathrm{~cm}$, $L^{*} \sim L_{1}^{*} \sim 10 \mathrm{~cm}, \omega^{*} \sim 1-30 \mathrm{mHz}$ and a typical surfactant with $\sigma_{0} \sim 70 \mathrm{dyn}^{-1}, \Pi\left(c_{0}^{*}\right) \sim$ $0-15$ dyn $\mathrm{cm}^{-1}$ and $c_{0}^{*} M\left(c_{0}^{*}\right) \sim 0-30 \mathrm{dyn}^{-1}$, we have

$L \sim L_{1} \sim 20, \quad \mathcal{G} \sim 10^{6}, \quad \mathcal{S} \sim 3.5 \times 10^{5}, \quad \mathcal{P} \sim 0-7.5 \times 10^{4}, \quad \omega \sim 0.15-4$.

Data on the surfactant diffusion coefficient are scarce, but surfactant diffusion usually has a negligible effect in most of the cases and we may assume that $\mathcal{D}$ is small. All these suggest the limit

$L \sim L_{1} \gg 1, \quad \mathcal{G} \gg L^{2}, \quad \mathcal{S} \gg 1, \quad \mathcal{D} \ll 1 \quad$ and either $\omega \ll 1$ or $\omega \sim 1$. 

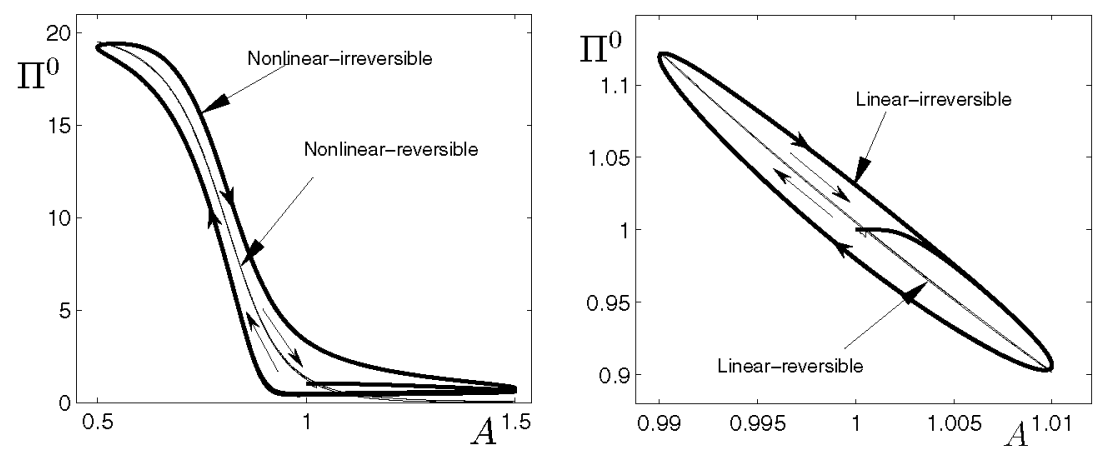

Figure 3. Typical Lissajous orbits for nonlinear (left) and linear (right) behaviors, for irreversible (thick solid lines, enclosing a non-zero area) and reversible (thin solid line, enclosing zero area) dynamics.

$\mathcal{P}$ instead varies in a wide range, depending on the surfactant concentration. Since $L \gg 1$, a long-wave (lubrication-like) approximation seems reasonable and will be derived in the next section, after pointing out the symmetries of the problem and its possible implications.

\subsection{Spatio-temporal symmetries, reversibility and Lissajous orbits}

As formulated, the problem (4)-(13) is invariant under the spatial reflection symmetry

$$
u \rightarrow-u, \quad x \rightarrow-x,
$$

but not under the temporal reflection symmetries

$$
t \rightarrow \pi / 2-t, \quad t \rightarrow 3 \pi / 2-t
$$

which are symmetries of the spatial domain according to (7) and (8) but, in principle, are broken by the time derivatives appearing in equations (5) and (6). In certain circumstances, some approximations of the problem are invariant under (19), which is an indication of reversible dynamics. For instance, runs with spatially uniform concentration are obviously reversible, and the concentration is inversely proportional to the area, which in turn is proportional to

$$
A(t)=1+a \sin t
$$

Namely, the concentration is given by

$$
c(t)=c(0) A(0) / A(t) \equiv c(0) /(1+a \sin t) .
$$

Since the experimental set up is designed expecting spatially uniform surfactant concentration, measurements are made only at one point of the spatial domain, namely at the axis of symmetry $x=0$. Results are given either as time series of the surface pressure at this point (denoted hereafter as $\Pi^{0}$ ) or using the so-called Lissajous orbits (a name taken from Celestial Mechanics), which are representations of $\Pi^{0}$ versus the rescaled area $A$, defined in (20). After a transient, the system exhibits periodic behavior, which means that the Lissajous orbit approaches a closed curve, as sketched in figure 3 (left). For irreversible dynamics, the lower and upper parts of this curve are different from each other and correspond to the compression and expansion stages of the cycle, respectively. 
For reversible runs, instead, the upper and lower branches collapse in a segment of a curve, which is covered twice in each compression/expansion cycle (see figure 3 (left)). On the other hand, if the forcing amplitude $a$ is sufficiently small, then the solid barriers oscillate harmonically around the positions $x= \pm L$ and a linear approximation applies. In this case, the flow variables and the perturbation of the surfactant concentration are invariant under the spatio-temporal symmetry

$$
(u, v, p, f, \Delta c) \rightarrow-(u, v, p, f, \Delta c), \quad t \rightarrow t+\pi .
$$

This symmetry can be identified in the Lissajous orbits (see figure 3 (right)), which are reflection symmetric around the unperturbed state, and also in the Fourier transform of the time series of the surface pressure, which exhibit odd sine modes only.

\section{The long-wave approximation}

As usual, the long-wave approximation relies on the assumption that all quantities are slowly varying in the $x$ direction (except near the solid barriers and the end-walls), which invoking the continuity equation (4) also implies that the vertical velocity is small,

$$
\partial_{x} \sim L^{-1} \partial_{y} \ll 1, \quad|v| \sim L^{-1}|u| \ll|u| .
$$

Thus, assuming (23) and rescaling variables as

$$
\xi=x / L, \quad \hat{u}=u /(\omega L), \quad \hat{v}=v / \omega, \quad \hat{p}=p /\left(\omega L^{2}\right) .
$$

The system of equations (5), (6), (9), (11) and (13) is reduced to

$$
\begin{aligned}
\partial_{\xi} \hat{u}+\partial_{y} \hat{v}=0, & \omega\left(\partial_{t} \hat{u}+\hat{u} \partial_{\xi} \hat{u}+\hat{v} \partial_{y} \hat{u}\right)=-\partial_{\xi} \hat{p}(\xi, t)+\partial_{y y} \hat{u} \quad \text { in } \quad-1<y<0, \\
& \hat{u}=0 \quad \text { at } \quad y=-1, \\
& \partial_{y} \hat{u}=-4 \hat{\mathcal{P}} \partial_{\xi} \Pi(c), \quad \partial_{t} c+\partial_{\xi}(\hat{u} c)=0 \quad \text { at } \quad y=0, \\
& \int_{-1}^{0} \hat{u} \mathrm{~d} y=0, \\
& \hat{u}= \pm X_{\mathrm{b}}^{\prime}(t) \quad \text { at } \quad y=0, \xi= \pm X_{\mathrm{b}}(t),
\end{aligned}
$$

where

$$
\hat{\mathcal{P}}=\mathcal{P} /\left(4 \omega L^{2}\right) .
$$

In this approximation we have neglected the free surface elevation, which is justified as follows. According to the non-dimensionalization above and the long-wave approximation, $|u| \sim \omega L,|p| \sim \omega L^{2}$ and $|v| \sim \omega$. These, in conjunction with (4), (12) and the assumption in (17) that $\mathcal{G} \gg L^{2}$, yield

$$
|f| \sim \omega L^{2} / \mathcal{G} \ll \omega \sim|v| .
$$

Thus, $\omega|f| \ll|v|$ and the boundary condition (10) reduces, after rescaling, to $\hat{v}=0$ at $y=0$, which integrating the continuity equation (4) in $-1<y<0$ yields the condition (28).

The system (25)-(28) applies in between the solid barriers, that is in the spatial region (7), excluding two capillary layers whose horizontal thickness is $O(\sqrt{\mathcal{S} / \mathcal{G}})$, as obtained from (12). Keeping in mind (16), this thickness is somewhat small (smaller than the layer depth, in dimensional terms), which means that it is quite small compared with $L$. Thus, the capillary layers can be neglected, and since these layers do not accumulate any significant amount 
of liquid, it follows that the horizontal velocity of the free surface at the solid barriers must coincide with the velocity of the solid barriers themselves, which is expressed by the boundary condition (29).

Equations (25)-(27) apply in the limit $\omega \sim 1$, which is relevant in some experimental runs (Kato and Kawaguchi 2012, Kobayashi and Kawaguchi 2013) and will be pursued elsewhere. Here, instead, we further simplify these equations, concentrating in the limit

$$
\omega \ll 1,
$$

which invoking (15) corresponds to slow motions and is the relevant limit in the reminder of the paper. In this limit, the second equation (25) reduces to $\partial_{y y} \hat{u}=\partial_{\xi} \hat{p}(\xi, t)$, which is readily integrated using (26) and the first boundary condition (27), to obtain

$$
\hat{u}=(y+1)^{2} \partial_{\xi} \hat{p} / 2-\left[\partial_{\xi} \hat{p}+4 \hat{\mathcal{P}} \partial_{\xi} \Pi(c)\right](y+1) .
$$

Imposing the first condition in (29) yields

$$
\partial_{\xi} \hat{p}=-6 \hat{\mathcal{P}} \partial_{\xi} \Pi(c),
$$

which substituted into (33) provides the horizontal velocity at the free surface, as

$$
\hat{u}=-\hat{\mathcal{P}} \partial_{\xi} \Pi(c) \quad \text { at } \quad y=0,
$$

meaning that the fluid dynamics is slaved to the surfactant concentration gradient, as anticipated. Substituting this into the second expressions in (27) and (29) provides the following evolution equation and boundary conditions for $c$ :

$$
\begin{aligned}
& \partial_{t} c=\hat{\mathcal{P}} \partial_{\xi}\left[c \partial_{\xi} \Pi(c)\right] \quad \text { in } \quad-X_{\mathrm{b}}(t)<\xi<X_{\mathrm{b}}(t), \\
& \hat{\mathcal{P}} \partial_{\xi} \Pi(c)=\mp X_{\mathrm{b}}^{\prime}(t) \quad \text { at } \quad \xi= \pm X_{\mathrm{b}}(t) .
\end{aligned}
$$

Equation (36) is a nonlinear diffusion equation. Similar equations (coupled with another equation accounting for the film height) have been derived by Borgas and Grotberg (1988) and repeatedly used in the context of dynamics of (insoluble) surfactant over thin liquid layers (Renardy 1996, Matar and Troian 1999, Dassaud et al 2005) and references therein). Note that the nonlinear diffusion term in equation (36) results from the interplay of Marangoni stress and the viscous diffusion of the bulk fluid (consistent with the lubrication approximation); the surfactant diffusion, instead, has been neglected and plays no role. On the other hand, the diffusivity $c \Pi^{\prime}(c)$ vanishes at both $c=0$ and as $c \rightarrow \infty$, which makes the equation singular in these two limits. Equations of this type are known as singular, nonlinear diffusion equations and because of the singularity at $c=0$ they may exhibit clean regions near the solid barriers, where the surfactant is completely absent (an example of this is given in figure 6 below).

Note also that the formulation (36), (37) preserves conservation of the total amount of surfactant, namely

$$
\frac{\mathrm{d}}{\mathrm{d} t} \int_{-X_{\mathrm{b}}(t)}^{X_{\mathrm{b}}(t)} c \mathrm{~d} \xi=0
$$

as obtained integrating (36) in the spatial domain $-X_{\mathrm{b}}(t)<\xi<X_{\mathrm{b}}(t)$, integrating by parts, and applying the boundary conditions (37). Equations (36) and (37) also preserve reflection symmetry since they are invariant under the action

$$
\xi \rightarrow-\xi \text {. }
$$


But no other symmetries are present. In particular, (36) is not invariant under (19), implying that the equation generally exhibits irreversible dynamics. It is to be noted that the surfactant concentration is spatially uniform (and the dynamics is reversible) in the limit

$$
\hat{\mathcal{P}} \gg 1
$$

which invoking the definition of $\hat{\mathcal{P}}$ in (30) corresponds to either very small forcing frequency or very large Marangoni elasticity. To this end, we note that in this limit the left hand side of equation (36) can be neglected and the resulting equation integrated, which yields

$$
c \partial_{\xi} \Pi(c)=K(t) \quad \text { in } \quad-X_{\mathrm{b}}(t)<\xi<X_{\mathrm{b}}(t) .
$$

The function $K(t)$ is determined by applying the boundary conditions (37), which leads to $K(t)=\mp c_{0}^{ \pm} X_{\mathrm{b}}^{\prime}(t) / \hat{\mathcal{P}}$, where $c_{0}^{ \pm}$denotes the surfactant concentrations at the barriers. Since $c_{0}^{ \pm} \geqslant 0$, the function $K(t)=0$ implying that $c \partial_{\xi} \Pi(c) \equiv c \Pi^{\prime}(c) \partial_{\xi} c=0$ in $-X_{\mathrm{b}}(t)<\xi<$ $X_{\mathrm{b}}(t)$, which means that $c=c(t)$. This function $c(t)$ is obtained from the conservation of the total amount of surfactant (38), to be

$$
c(t)=\frac{c(0) X_{\mathrm{b}}(0)}{X_{\mathrm{b}}(t)},
$$

which implies that the dynamics is reversible in this limit.

\section{Results}

In this section we show the results obtained from the numerical integration of the system (36) and (37) for several representative cases. In order to numerically integrate the problem, equations (36) and (37) are first rewritten in a fixed spatial domain, using the spatial variable $\eta=\xi / X_{\mathrm{b}}(t)$ and then discretized using a finite volume scheme, in a strong conservation form, which allows obtaining robust results even in cases in which the surfactant concentration is very small in a part of the domain (see below), without adding any extra diffusivity to smooth out singularities. The resulting set of ordinary differential equations is integrated using standard MatLab@ routines. In all numerical runs, the initial condition for the surfactant concentration is spatially uniform with a value that corresponds to the semidiluted regime, and we assume a trough of $50 \mathrm{~cm}$ long and $0.4 \mathrm{~cm}$ deep, with the oscillatory barriers oscillating at a constant frequency $5.4 \mathrm{mHz}$ (the parameters of the experimental set-up used by Hilles et al 2006, 2007). The results will be concerned with PVAc and PODA, using the approximations for $\Pi^{*}$ and $M^{*}$ versus $c^{*}$ provided in figure 2 (right) and considering qualitative comparison with some experiments in the literature. Quantitative comparison is not intended because of the lack of quantitative information of some details of the experimental set ups. For instance, the depth of the fluid layer (which is essential in the approximation above) is never provided, seemingly because it is expected that the fluid below the surfactant monolayer plays no role. In other words, new experiments are necessary to conveniently account for the fluid dynamics.

We first compare with the experimental results obtained by Hilles et al (2006) for a PVAcmonolayer. As shown in figure 4, the theoretical and experimental results are qualitatively similar and clearly nonlinear since linear theory would provide a harmonic sinusoidal response. Figure 4 also shows that the dynamics is only slightly irreversible, since the time series approximately shows the symmetries (19); note that the non-dimensional values $t=\pi / 2$ and $3 \pi / 2$ correspond to $t^{*}=46.3$ and 138.9 , respectively, in figure 4 . The almost irreversible dynamics is due to the small forcing frequency; at larger frequency, irreversibility would have been more apparent. 

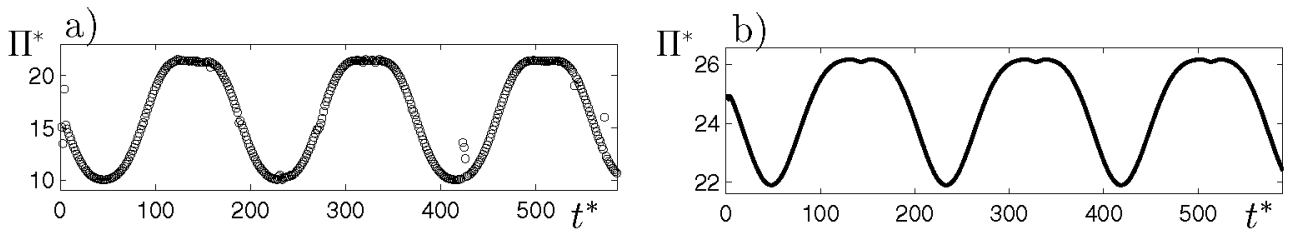

Figure 4. Time series of the surface pressure at the center of the trough for a PVAc-monolayer. (a) Experimental results obtained by Hilles et al (2006), for initial concentration $c_{0}^{*}=1$ and forcing amplitude $a=0.37$ (courtesy of R G Rubio). (b) Results obtained from the numerical integration of equations (36) and (37) for initial condition $c_{0}^{*}=1.5$, forcing amplitude $a=0.6$ and $\hat{\mathcal{P}}=27.1$.
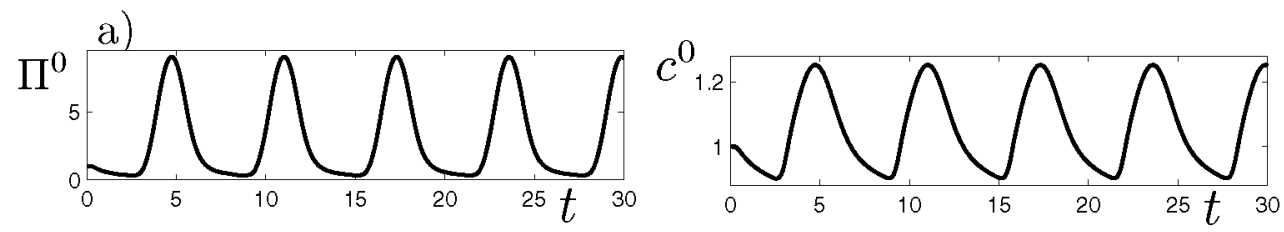

b)
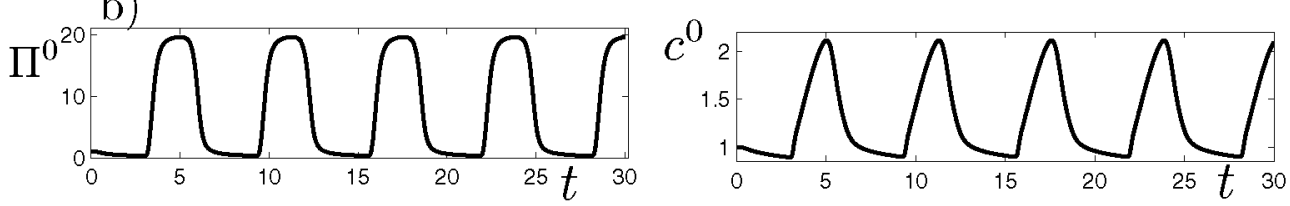

Figure 5. Time series of the surface pressure, $\Pi^{0}$, and of the surfactant concentration $c^{0}$, both measured at the center of the trough. Results for a PODA-monolayer, obtained from the numerical integration of (36) and (37), with initial condition $c_{0}^{*}=1, \hat{\mathcal{P}}=0.7$, and forcing amplitude (a) $a=0.1$ and (b) $a=0.5$.

More clearly irreversible dynamics were obtained by Hilles et al (2007) (see figure 4 of that article) using a PODA-monolayer. These measurements which compare qualitatively well with the theoretical runs in figure 5 , where the irreversibility is clear, namely the symmetries (19) are absent. This figure also shows that the nonlinear behavior appears in these monolayers for rather small forcing amplitudes, which is due to the fairly steep behavior of the curve $\Pi^{*}$ versus $c^{*}$ (see figure 2). As expected from the theory above, the influence from the dynamics of the fluid bulk on the surface dynamics of the monolayer gives rise to spatially non-uniform surfactant concentration distributions, as also shown in figures 5 (right) and 6. An interesting dynamic behavior of what may also be found as a consequence of this effect, is illustrated as well in figure 6 , which shows, during one period, the profiles of the surfactant concentration distributions versus the variable $\eta=\xi /(1+a \sin t)$ (a scaled variable to show all domain in the interval $[-1,1])$. As can be seen, initially the surfactant concentration is nearly uniform along the entire trough, but, after some time, the surfactant starts to concentrate away from the solid barriers, creating two small areas close to the barriers with zero surfactant concentration. As the time passes, that process is reversed until the initial state is again reached.

The irreversibility may also be observed through the Lissajous orbits, exhibiting hysteresis cycles when these orbits enclose a region of non-zero area. Figure 7 shows the Lissajous orbits for a PVAc-monolayer, for four different initial conditions. It is worth mentioning that this behavior is entirely due to the influence of bulk fluid dynamics on the 

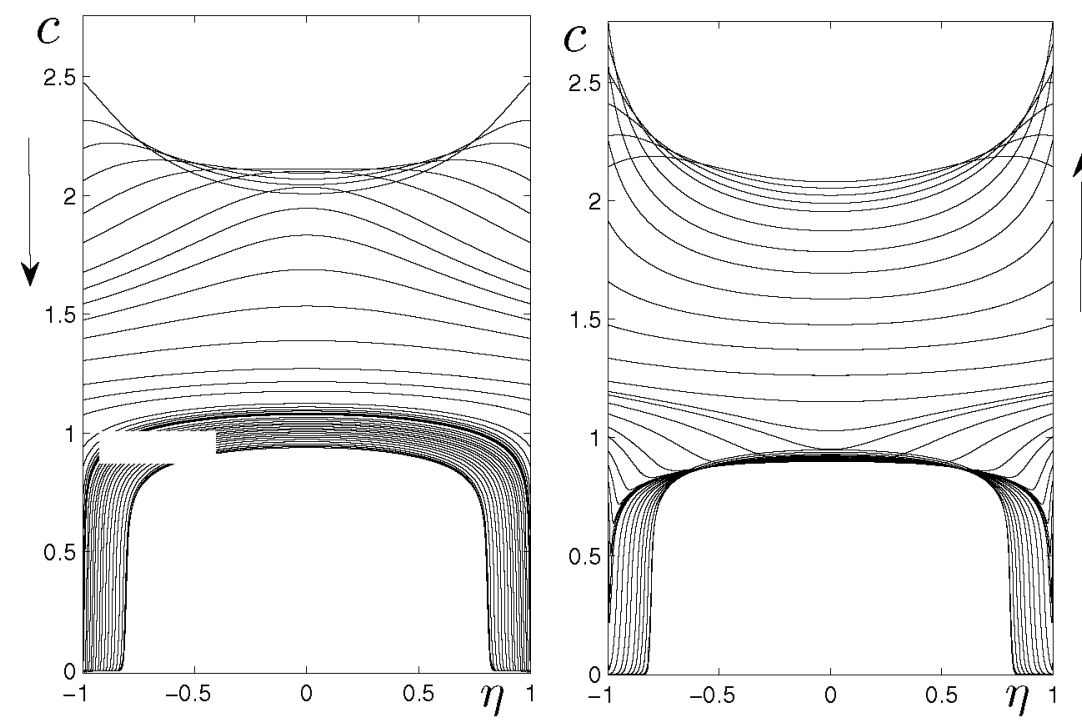

Figure 6. Profiles of the surfactant concentration distributions versus the variable $\eta=$ $\xi /(1+a \sin t)$, during one period, for a PODA-monolayer. These results are obtained from numerical integration of (36) and (37) with initial condition $c_{0}^{*}=1$, forcing amplitude $a=0.3$, and $\hat{\mathcal{P}}=0.7$. The arrows indicate the direction of increasing time.
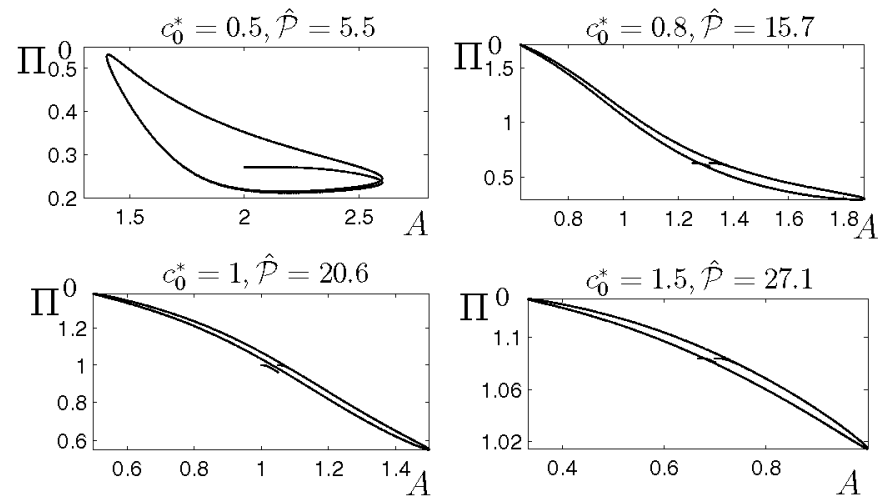

Figure 7. Lissajous orbits giving the surface pressure $\Pi^{0}$ versus the area in between the barriers $A$. Results for a PVAc-monolayer, obtained from numerical integration of (36) and (37) for the indicated initial conditions $c_{0}^{*}$ and forcing amplitude $a=0.3$.

monolayer while, in most experimental articles, irreversibility is attributed to non-equilibrium effects related to physico-chemical properties. Figure 7 also shows that there are qualitative differences in the shape of the orbits in the distinct regimes, which is imposed by the concave and convex shapes of the equilibrium curve (see figure 2).

\section{Conclusions}

A long-wave approximation has been derived to describe the dynamics of Langmuir monolayers when these are compressed/expanded periodically. The resulting equations have 
been further simplified in the limit of small forcing frequency, to obtain a nonlinear diffusion equation which describes the surfactant concentration dynamics.

In this limit, the fluid dynamics are slaved to the surfactant concentration gradient and the nonlinear diffusion term in the equation results from the interplay of Marangoni stress and the restoring effect of the fluid dynamics in the bulk, which is consistent with the lubrication approximation in the long-wave limit that is considered. The conclusion is that the fluid dynamics in the bulk is an important source of irreversibility, which could explain some of the irreversible experimental observations. Ignoring any additional non-equilibrium effects, the formulation provides results (see figures 4-7) that compare qualitatively well with their experimental counterparts. Non-equilibrium effects can of course be present too, but these should be analyzed as a source of irreversibility in conjunction with (not in place of) the fluid dynamics. There are additional fluid dynamic effects that have been neglected in this paper, such as the surface viscosity, the free surface deformation, the dynamics of the menisci attached to both the barriers and the surface tension measuring plate located in the symmetry plane, and the pressure drop due to viscous dissipation in the bulk phase across the barriers. Depending on the specific values of the parameters, these effects could be non-negligible and influence the response of the system as well. In particular:

- the analysis of the free surface deformation requires one to also consider the fluid dynamics in the outer regions, outside the barriers, which may promote differences in the mean free surface elevation in the inner and outer regions;

- the menisci dynamics could promote contact line motion that in turn would affect the surface tension measurements in the plate.

All these are the object of our current research.

\section{Acknowledgments}

This research was partially supported by the Spanish Ministry of Science and Innovation, under grant TRA2010-18054.

\section{References}

Borgas M S and Grotberg J B 1988 Monolayer flow on a thin film J. Fluid Mech. 193 151-70

Couder Y, Chomaz J M and Rabaud M 1989 On the hydrodynamics of soap films Physica D 37 384-405

Dassaud A D, Matar O K and Troian S M 2005 Spreading characteristics of an insoluble surfactant film on a thin liquid layer: comparison between theory and experiment $J$. Fluid Mech. 544 23-51

Fainerman V B, Lylyk S V, Aksenenko E V, Petkov J T, Yorke J and Miller R 2010 Surface tension isotherms, adsorption dynamics and dilational visco-elasticity of sodium dodecyl sulphate Colloid Surf. A $3548-15$

Herrada M A, Montanero J M and Vega J M 2011 The effect of surface shear viscosity on the damping of oscillations of millimetric liquid bridges Phys. Fluids $\mathbf{2 3} 082102$

Hilles H, Monroy F, Bonales L J, Ortega F and Rubio R G 2006 Fourier transform rheology of polymer Langmuir monolayers: analysis of the nonlinear and plastic behaviors Adv. Colloid Interface Sci. $12267-77$

Hilles H, Maestro A, Monroy F, Ortega F, Rubio R G and Velarde M G 2007 Polymer monolayers with a small viscoelastic linear regime: equilibrium and rheology of poly(octadecyl acrylate) and poly(vinyl stearate) J. Chem. Phys. 126124904

Kato S and Kawaguchi M 2012 Surface dilatational moduli of poly (ethylene oxide), poly (methyl methacrylate) and their bended films $J$. Colloid Interface Sci. 384 87-93 
Kobayashi T and Kawaguchi M 2013 Surface dilatational moduli of latex-particle monolayers spread in air-water interface J. Colloid Interface Sci. 390 147-50

Langevin D and Bergeron V 1996 Thin-film forces in foam films containing anionic polyelectrolyte and charged surfactants Langmuir 12 1550-6

Matar O K and Troian S M 1999 Spreading of a surfactant monolayer on a thin liquid film: onset and evolution of digitated structures Chaos 9 141-53

Miller C A and Scriven L E 1968 The oscillations of a fluid droplet immersed in another fluid $J$. Fluid Mech. 32 417-35

Renardy M 1996 On an equation describing the spreading of surfactants on thin films Nonlinear Anal. TMA 26 1207-19

Sagis L M C 2011 Dynamic properties of interfaces in soft matter: experiments and theory Rev. Modern Phys. 83 1367-403 